Service social

Guide d'intervention de groupe auprès des parents d'adolescents, par Michel Y. Ménard et Camile Spain, Pierrefonds, C.L.S.C. Pierrefonds, 1989, 84 pages.

\title{
Jocelyn Lindsay
}

Volume 38, numéro 1, 1989

Aspects psychosociaux du Sida

URI : https://id.erudit.org/iderudit/706432ar

DOI : https://doi.org/10.7202/706432ar

Aller au sommaire du numéro

Éditeur(s)

École de service social de l'Université Laval

ISSN

1708-1734 (numérique)

Découvrir la revue

Citer ce compte rendu

Lindsay, J. (1989). Compte rendu de [Guide d'intervention de groupe auprès des parents d'adolescents, par Michel Y. Ménard et Camile Spain, Pierrefonds, C.L.S.C. Pierrefonds, 1989, 84 pages.] Service social, 38(1), 140-141.

https://doi.org/10.7202/706432ar d'utilisation que vous pouvez consulter en ligne.

https://apropos.erudit.org/fr/usagers/politique-dutilisation/ 
particuliers pour ensuite définir les aptitudes qu'il convient de développer pour le rendre aisément accessible et, même, pour le généraliser " ${ }^{1}$

Germain Trottier

École de service social,

Université Laval.

\section{Notes et références}

* Le masculin est utilisé sans discrimination et dans le seul but d'alléger le texte.

1 BLONDIN, René, Le bonheur possible, Montréal, Les Éditions de l'homme, 1983, p. 11.

Guide d'intervention de groupe auprès des parents d'adolescents, par Michel Y. Ménard et Camile SPAIN, Pierrefonds, C.L.S.C. Pierrefonds, 1989, 84 pages.

D'une façon détaillée, ce guide expose le programme Parents d'adolescents bien branchés mis en place au C.L.S.C. Pierrefonds depuis 1981. Ce programme a pour but de "permettre aux parents d'adolescents d'accroître leur compétence parentale pour améliorer les relations parents/adolescents» (p. 12). Il en découle des objectifs aux niveaux cognitif, affectif et comportemental, par exemple améliorer ses connaissances sur l'adolescence, développer une prise de conscience de ses attitudes ou maîtriser une méthode de résolution des conflits.

Mettant en évidence les besoins des parents d'adolescents dans leur tâche d'éducation, les auteurs souhaitent que les personnes qui veulent travailler en groupe auprès de cette population trouvent dans cet ouvrage des idées, des exercices et même une méthode globale d'animation d'une rencontre thématique.

Quittant, tel qu'il est mentionné dans la préface, le "sentier officiel des méthodes à idéologie unique ", les auteurs ont trouvé leurs sources d'inspiration dans des expériences faites au Bureau de Consultation-Jeunesse, à la Commission des Écoles Catholiques de Montréal et au C.L.S.C. Centre-Sud. Au plan théorique, ils se réfèrent surtout au service social des groupes (groupe structuré d'éducation et modèle du courant central).

Après une présentation des activités de la phase pré-groupe, le guide se divise en dix parties correspondant aux thèmes de chacune des rencontres: le contrat, la psychologie de l'adolescence, l'initiation à la communication, l'écoute empathique, l'expression des sentiments, les drogues et la rivalité fraternelle, la sexualité, la résolution des conflits, la motivation scolaire et les loisirs, ainsi que l'honnêteté et l'évaluation des acquis. On retrouve aussi, pour chacune de ces rencontres, une procédure identique: une présentation du contenu de la rencontre, un exercice de réchauffement, un retour sur l'exercice à la maison, 
quelques activités (exposé, jeu de rôle, etc.) présentées de façon détaillée, des exercices à faire chez soi, un retour sur la rencontre et des annexes complémentaires.

Il est très heureux que les auteurs aient compilé dans un tel guide un savoirfaire qui s'étend sur plusieurs années et qui pourra profiter à de nombreux intervenants et intervenantes œuvrant autour de cette problématique. Également, il est rare que des auteurs, fussent-ils d'excellents praticiens, décrivent leur intervention avec autant de minutie. Le lecteur intéressé à approfondir le développement de ce programme ou ses dimensions théoriques consultera avec avantage un article de la seconde auteure, paru dans un précédent numéro de Service Social (vol. 35, no 3, 1986, pp. 352-365).

Ce type d'ouvrage peut avoir, au plan de l'utilisation, le défaut de sa qualité : il ne faut jamais oublier qu'un tel document n'est pas un manuel de recettes, mais plutôt le résultat tangible et écrit d'une longue expérience auprès des parents d'adolescents. Dans cette perspective, le lecteur y trouvera son profit, et largement.

Jocelyn LINDSAY

École de service social,

Université Laval. 